\title{
AN ANALYTICITY RESULT FOR THE DEPENDENCE OF MULTIPLE EIGENVALUES AND EIGENSPACES OF THE LAPLACE OPERATOR UPON PERTURBATION OF THE DOMAIN
}

\author{
PIER DOMENICO LAMBERTI and MASSIMO LANZA DE CRISTOFORIS \\ Dipartimento di Matematica Pura ed Applicata, Università di Padova, Via Belzoni 7, 35131, Padova, Italy \\ e-mail:lamberti@math.unipd.it,mldc@math.unipd.it
}

(Received 16 February, 2000; revised 8 May, 2001)

\begin{abstract}
In this paper, we consider the dependence of the Dirichlet eigenvalues and eigenspaces of the Laplace operator upon perturbation of the domain of definition. We prove that the dependence of a certain eigenvalue and of the corresponding eigenspace is analytic on the set of perturbations that leave the multiplicity constant.
\end{abstract}

2000 Mathematics Subject Classification. 35P05.

1. Introduction. For a given regular, connected, bounded open subset $\Omega$ of $\mathbb{R}^{n}$, we consider the Dirichlet eigenvalue problem

$$
\begin{cases}-\Delta u=\lambda u & \text { in } \Omega, \\ u=0 & \text { on } \partial \Omega\end{cases}
$$

for $\lambda \in \mathbb{R}, u$ in the Schauder space $C^{k, \alpha}(\mathrm{cl} \Omega)$ of the $k$-times continuously differentiable functions of $\operatorname{cl} \Omega$ to $\mathbb{R}$, with $\alpha$-Hölder continuous $k$-th order derivatives, for $k \geq 2, \alpha \in] 0,1[$. It is well known that problem (1.1) has an increasing sequence of eigenvalues, that we write as $0<\lambda_{1}<\lambda_{2} \leq \ldots \leq \lambda_{j} \leq \ldots$, where each eigenvalue is repeated as many times as its multiplicity. If $\phi$ is a diffeomorphism of $\operatorname{cl} \Omega$ onto $\operatorname{cl} \phi(\Omega)$ of class $\left(C^{k, \alpha}(\mathrm{cl} \Omega)\right)^{n}$, then $\phi(\Omega)$ is again a sufficiently regular bounded open domain of $\mathbb{R}^{n}$, and it makes sense to consider the Dirichlet eigenvalues $\left\{\lambda_{j}[\phi]\right\}_{j \in \mathbb{N} \backslash\{0\}}$ of $-\Delta$ in the 'perturbed' domain $\phi(\Omega)$.

Several authors have investigated the problem of establishing the regularity of the maps which take a perturbed domain, here $\phi(\Omega)$, to the spectrum of $-\Delta$ in $\phi(\Omega)$, here $\left\{\lambda_{j}[\phi]\right\}_{j \in \mathbb{N} \backslash\{0\}}$. For a treatment of this problem, we refer to Chow and Hale [2], Desaint and Zolésio [5] and Henry [9].

It is well known that the maps $\phi \mapsto \lambda_{j}[\phi]$ are continuous (cf. Courant and Hilbert [3].) Cox [4] has shown a Lipschitz continuity result for the dependence of $\lambda_{j}[\phi]$ upon $\phi$. It is also known that if a certain eigenvalue $\lambda_{j}[\tilde{\phi}]$ relative to the domain $\tilde{\phi}(\Omega)$ has multiplicity one, then $\lambda_{j}[\cdot]$, as well as a suitably normalized eigenvector relative to $\lambda_{j}[\cdot]$, depend real analytically upon $\phi$ in a neighborhood of $\tilde{\phi}$ (cf. Henry [9], Prodi [21]). For related results, see also Chow and Hale [2], Desaint and Zolésio [5], [6] and Gekeler [7]. 
In general, if the multiplicity of $\lambda_{j}[\tilde{\phi}]$ is greater than one, we do not expect that $\lambda_{j}[\phi]$ depends analytically on $\phi$ around $\tilde{\phi}$. However, it makes sense to consider the set of perturbations, say $\mathcal{M}_{k, \alpha, j, m}$, of those $\phi$ 's for which $\lambda_{j}[\phi]$ has multiplicity $m$, and one may question whether the function $\lambda_{j}[\cdot]$, as well as the eigenspace relative to $\lambda_{j}[\cdot]$, depend with a certain degree of regularity on $\phi \in \mathcal{M}_{k, \alpha, j, m}$.

We note that Kong, Wu and Zettl [10] have proved some results related in spirit to ours. Namely, they have considered certain boundary-value problems for ordinary differential equations depending on a parameter, and they have proved differentiability theorems for eigenvalues of multiplicity higher than one upon variation of the parameter, under the assumption that locally the multiplicity of the eigenvalue does not change as the parameter is perturbed.

In this paper, we prove that if the multiplicity $m$ of $\lambda_{j}[\tilde{\phi}]$ is greater than one, then the restriction of $\lambda_{j}[\cdot]$ to $\mathcal{M}_{k, \alpha, j, m}$, and the function which takes $\phi \in \mathcal{M}_{k, \alpha, j, m}$ to a suitably chosen projection operator $P_{\phi, j}$ of the space for $u$ onto the eigenspace relative to $\lambda_{j}[\cdot]$ can be extended real-analytically in a neighborhood of $\tilde{\phi}$ to a function $\hat{\lambda}_{\tilde{\phi}, j}[\cdot]$, and to a function $\hat{P}_{\tilde{\phi}, j}[\cdot]$ respectively. It should be mentioned however, that if $\phi$ does not belong to $\mathcal{M}_{k, \alpha, j, m}$, then we cannot expect, in general, that $\hat{\lambda}_{\tilde{\phi}, j}[\phi]$ and $\hat{P}_{\tilde{\phi}, j}[\phi]$ coincide with $\lambda_{j}[\phi]$ and $P_{\phi, j}$, respectively.

The idea of the proof is to set up a suitable functional equation involving the eigenvalues, the function $\phi$, and the projection operators associated to the eigenspaces, and to use the Implicit Function Theorem. Our methods differ completely from the classical methods used to study the analytic dependence of the eigenvalues of a real or complex parameter dependent family of compact self adjoint linear operators in Hilbert space, which could instead be used to deduce analyticity results for the dependence of $\lambda_{j}[\gamma(t)]$ upon $t$, when $\{\gamma(t)\}_{t \in \mathbb{R}}$ is a one parameter real analytic family of domain diffeomorphisms.

It should be noted that Lupo and Micheletti [15] have proved that the set of $\phi$ 's of class $C^{3}$ which preserve the multiplicity $m=2$ of a certain eigenvalue is locally a manifold of codimension $\frac{m(m+1)}{2}-1$ in $C^{3}$, and have given a sufficient condition for the same result to hold for $m>2$. (See also Lupo and Micheletti [16], [17] for related results.) As we shall see in a forthcoming paper, the same result can be proved for $\phi$ 's of class $C^{k, \alpha}$ by exploiting the methods of this paper.

2. Technical preliminaries and notation. We denote the norm on a (real) normed space $\mathcal{X}$ by $\|\cdot\|_{\mathcal{X}}$. Let $\mathcal{X}$ and $\mathcal{Y}$ be normed spaces. We equip the product space $\mathcal{X} \times \mathcal{Y}$ with the norm $\|\cdot\|_{\mathcal{X} \times \mathcal{Y}} \equiv\|\cdot\|_{\mathcal{X}}+\|\cdot\|_{\mathcal{Y}}$, while we use the Euclidean norm for $\mathbb{R}^{n}$. By $\mathcal{L}(\mathcal{X}, \mathcal{Y})$ we denote the normed space of the continuous linear maps of $\mathcal{X}$ to $\mathcal{Y}$ equipped with the topology of uniform convergence on the unit sphere of $\mathcal{X}$. $\mathcal{B}(\mathcal{X} \times \mathcal{Y}, \mathcal{Z})$ denotes the normed space of the continuous bilinear maps of $\mathcal{X} \times \mathcal{Y}$ to the normed space $\mathcal{Z}$, and is equipped with the topology of uniform convergence on the cross product of the unit sphere of $\mathcal{X}$ and of the unit sphere of $\mathcal{Y}$. For standard definitions of calculus in normed spaces, we refer to Prodi and Ambrosetti [22]. Furthermore, $\mathbb{N}$ denotes the set of natural numbers including 0 . Throughout the paper, $n$ is an element of $\mathbb{N} \backslash\{0\}$. The inverse function of an invertible function $f$ is denoted $f^{(-1)}$, as opposed to the reciprocal of a complex-valued function $g$, or the inverse of a matrix $A$, which are denoted $g^{-1}$ and $A^{-1}$, respectively. A dot '. ' denotes the inner product in $\mathbb{R}^{n}$, or the matrix product between matrices with real entries. Let $A$ be a matrix. Then $A^{t}$ denotes the transpose matrix of $A$, and $\operatorname{tr} A$ denotes the 
trace of $A$. The set of $r \times r$ matrices with real entries is denoted by $M_{r}(\mathbb{R})$. Let $E \subseteq \mathbb{R}^{n}$. Then $\operatorname{cl} E$ denotes the closure of $E$. For all $R>0, x \in \mathbb{R}^{n},|x|$ denotes the Euclidean modulus of $x$ in $\mathbb{R}^{n}$, and $B(x, R)$ denotes the ball $\left\{y \in \mathbb{R}^{n}:|x-y|<R\right\}$. Let $\Omega$ be an open subset of $\mathbb{R}^{n}$. The space of $k$ times continuously differentiable realvalued functions on $\Omega$ is denoted by $C^{k}(\Omega)$. Let $f \in\left(C^{k}(\Omega)\right)^{n}$. The $s$-th component of $f$ is denoted $f_{s}$, and $D f$ denotes the gradient matrix $\left(\frac{\partial f_{s}}{\partial x_{l}}\right)_{s, l=1, \ldots, n}$. Let $\eta \equiv$ $\left(\eta_{1}, \ldots, \eta_{n}\right) \in \mathbb{N}^{n},|\eta| \equiv \eta_{1}+\ldots+\eta_{n}$. Then $D^{\eta} f$ denotes $\frac{\partial^{|\eta|} \mid}{\partial x_{1}^{\eta 1} \ldots \partial x_{n}^{\eta} n}$. The subspace of $C^{k}(\Omega)$ of those functions $f$ such that $f$ and its derivatives $D^{\eta} f$ of order $|\eta| \leq k$ can be extended with continuity to $\operatorname{cl} \Omega$ is denoted by $C^{k}(\operatorname{cl} \Omega)$. Let $\Omega$ be a bounded open subset of $\mathbb{R}^{n}$. $C^{k}(\mathrm{cl} \Omega)$ equipped with the norm $\|f\|_{k} \equiv \sum_{|\eta|<k} \sup _{\mathrm{cl} \Omega}\left|D^{\eta} f\right|$ is a Banach space. The subspace of $C^{k}(\operatorname{cl} \Omega)$ whose functions have $k$-th order derivatives that are Hölder continuous with exponent $\alpha \in(0,1]$ is denoted $C^{k, \alpha}(\mathrm{cl} \Omega)$, (cf. e.g. Gilbarg and Trudinger [8].) Let $E \subseteq \mathbb{R}^{n}$. Then $C^{k, \alpha}(\mathrm{cl} \Omega, E)$ denotes $\left\{f \in\left(C^{k, \alpha}(\mathrm{cl} \Omega)\right)^{n}: f(\operatorname{cl} \Omega) \subseteq E\right\} . C^{k, \alpha}\left(\operatorname{cl} \Omega, M_{r}(\mathbb{R})\right)$ denotes the space of functions of $\mathrm{cl} \Omega$ to $M_{r}(\mathbb{R})$, whose components are of class $C^{k, \alpha}$. If $f \in C^{0, \alpha}(\mathrm{cl} \Omega)$, then its Hölder quotient $|f|_{\alpha}$ is defined as $\left\{\frac{|f(x)-f(y)|}{|x-y|^{\alpha}}: x, y \in \operatorname{cl} \Omega, x \neq y\right\}$. The space $C^{k, \alpha}(\operatorname{cl} \Omega)$, equipped with its usual norm $\|f\|_{k, \alpha}=\|f\|_{k}+\sum_{|\eta|=k}\left|D^{\eta} f\right|_{\alpha}$, is well known to be a Banach space. We say that a bounded open subset is of class $C^{k}$ or of class $C^{k, \alpha}$, if it is a manifold with boundary imbedded in $\mathbb{R}^{n}$ of class $C^{k}$ or $C^{k, \alpha}$, respectively.

We summarize in the following statement some known properties of the Schauder spaces that we need in the sequel (cf. e.g. Gilbarg and Trudinger [8], and [12, Section 2]).

Lemma 2.1. Let $k \in \mathbb{N}, \alpha, \beta \in] 0,1]$. Let $\Omega$ be a bounded connected open subset of $\mathbb{R}^{n}$ of class $C^{1}$.

(i) The pointwise product is continuous in $C^{k, \alpha}(\mathrm{cl} \Omega)$.

(ii) $C^{k+1}(\mathrm{cl} \Omega)$ is continuously imbedded in $C^{k, 1}(\mathrm{cl} \Omega)$.

(iii) If $\alpha>\beta$, then $C^{k, \alpha}(\mathrm{cl} \Omega)$ is compactly imbedded in $C^{k, \beta}(\mathrm{cl} \Omega)$.

(iv) If $\left\{f_{l}\right\}_{l \in \mathbb{N}}$ is a bounded sequence of $C^{k, \alpha}(\mathrm{cl} \Omega)$, that converges pointwise to some $f \in C^{0}(\mathrm{cl} \Omega)$, then $f \in C^{k, \alpha}(\operatorname{cl} \Omega)$ and $\left\{f_{l}\right\}_{l \in \mathbb{N}}$ converges to $f$ in $C^{k, \beta}(\mathrm{cl} \Omega)$, for all $\beta \in] 0, \alpha[$.

As we have announced in the introduction, we shall parametrize our domains by means of diffeomorphisms $\phi \in C^{k, \alpha}\left(\operatorname{cl} \Omega, \mathbb{R}^{n}\right)$. To apply our methods, we need to show that the set of such $\phi$ 's is open. Thus we introduce the following variant of $[\mathbf{1 3}$, Lemma 5.2] and [14, Lemma 2.2].

Lemma 2.2. Let $\Omega$ be a bounded connected open subset of $\mathbb{R}^{n}$ of class $C^{1}$. Then the following statements hold.

(i) The set $\mathcal{A} \equiv\left\{\phi \in C^{1}\left(\operatorname{cl} \Omega, \mathbb{R}^{n}\right): \phi\right.$ is injective, $\left.\operatorname{det} D \phi(x) \neq 0, \forall x \in \operatorname{cl} \Omega\right\}$ is open in $C^{1}\left(\mathrm{cl} \Omega, \mathbb{R}^{n}\right)$.

(ii) If $\phi \in \mathcal{A}$, then $\partial \phi(\mathrm{cl} \Omega)=\phi(\partial \Omega)=\partial \phi(\Omega)$, and $\phi(\Omega)$ is the interior of $\phi(\mathrm{cl} \Omega)$. The map $\phi$ is a homeomorphism of $\operatorname{cl} \Omega$ onto $\operatorname{cl} \phi(\Omega)$.

We summarize in the following Lemma the properties of the composition and of the inversion of functions in Schauder spaces that we need in the sequel. For a proof, we refer to [12, Lemmas 3.1, 4.26, and Theorem. 4.28]. 
Lemma 2.3. Let $k \in \mathbb{N} \backslash\{0\}, \alpha \in] 0,1]$. Let $\Omega, \Omega_{1}$ be open bounded connected subsets of class $C^{1}$ of $\mathbb{R}^{n}$. Then the following statements hold.

(i) If $(\phi, \psi) \in C^{k, \alpha}\left(\mathrm{cl} \Omega_{1}\right) \times C^{k, \alpha}\left(\mathrm{cl} \Omega, \operatorname{cl} \Omega_{1}\right)$, then $\phi \circ \psi \in C^{k, \alpha}(\mathrm{cl} \Omega)$.

(ii) If $\phi \in C^{k, \alpha}\left(\operatorname{cl} \Omega, \mathbb{R}^{n}\right) \cap \mathcal{A}$, then the inverse function $\phi^{(-1)} \in C^{k, \alpha}\left(\operatorname{cl} \phi(\Omega), \mathbb{R}^{n}\right)$.

Next, we have the following result.

Lemma 2.4. Let $k \in \mathbb{N} \backslash\{0\}, \alpha \in] 0,1]$. Let $\Omega$ be an open bounded connected subset of $\mathbb{R}^{n}$ of class $C^{k, \alpha}$. Let $\phi \in C^{k, \alpha}\left(\operatorname{cl} \Omega, \mathbb{R}^{n}\right) \cap \mathcal{A}$. Then $\phi(\Omega)$ is of class $C^{k, \alpha}$.

Proof. Since $\Omega$ is of class $C^{k, \alpha}$, it is well known that each $\phi \in C^{k, \alpha}\left(\operatorname{cl} \Omega, \mathbb{R}^{n}\right)$ admits an extension $\Phi$ of class $C^{k, \alpha}$ in a ball $\operatorname{cl} B(0, R)$ containing $\operatorname{cl} \Omega$ in the interior. By applying the Inverse Function Theorem to the function $\Phi$ at the points of $\partial \Omega$, and by using the local charts of $\partial \Omega$, one can easily construct local charts around the points of $\partial \phi(\Omega)$.

We collect in the following Theorem a few well-known facts on the eigenvalues of the Laplace operator.

Theorem 2.5. Let $k \in \mathbb{N} \backslash\{0,1\}, \alpha \in] 0,1[$. Let $\Omega$ be an open bounded connected subset of $\mathbb{R}^{n}$ of class $C^{k, \alpha}$. Let $\phi \in C^{k, \alpha}\left(\mathrm{cl} \Omega, \mathbb{R}^{n}\right) \cap \mathcal{A}$. Then the boundary value problem

$$
\begin{cases}-\Delta v=\lambda v & \text { in } \phi(\Omega), \\ v=0 & \text { on } \partial \phi(\Omega)\end{cases}
$$

has a nontrivial solution $v \in C^{k, \alpha}(\operatorname{cl} \phi(\Omega))$ if and only if $\lambda$ is a term of an increasing sequence $\left\{\lambda_{j}[\phi]\right\}_{j \in \mathbb{N} \backslash\{0\}}$ in $] 0,+\infty\left[\right.$. For each $\lambda \in\left\{\lambda_{j}[\phi]: j \in \mathbb{N} \backslash\{0\}\right\}$, problem (2.6) has a finite dimensional space of solutions. We call the dimension of this space the multiplicity of the eigenvalue $\lambda$, and we agree to repeat each eigenvalue in the sequence $\left\{\lambda_{j}[\phi]\right\}_{j \in \mathbb{N} \backslash\{0\}}$ as many times as its multiplicity. For each fixed $j \in \mathbb{N} \backslash\{0\}$, the map $\lambda_{j}[\cdot]$ of $C^{k, \alpha}\left(\mathrm{cl} \Omega, \mathbb{R}^{n}\right) \cap \mathcal{A}$ to $\mathbb{R}$ that takes $\phi$ to $\lambda_{j}[\phi]$ is continuous.

For the proof of the first part of the statement, we refer to Nečas [20, Theorem 3.1, p. 135] and to Gilbarg and Trudinger [8, Theorem 6.19, p. 111 and Theorem 8.37 , p. 214]. For the statement concerning the continuity of the eigenvalues, we refer to Courant and Hilbert [3, Theorem 8, p. 419].

We close the present section by collecting some known properties of elementary operators in the following Lemma. We note that throughout the paper 'analytic' means 'real analytic'. For the definition of analytic operator, we refer to Prodi and Ambrosetti [22, p. 89].

Lemma 2.7. Let $r, k \in \mathbb{N}, r>0, \alpha \in] 0,1]$. Let $\Omega$ be a bounded connected open subset of $\mathbb{R}^{n}$ of class $C^{1}$. Let $F^{-1}$ be the inverse matrix of (an invertible) $F \in C^{k, \alpha}\left(\mathrm{cl} \Omega, M_{r}(\mathbb{R})\right)$.

(i) The pointwise matrix product, which reduces to the pointwise product of functions when $r=1$, is bilinear and continuous and henceforth analytic from the space $C^{k, \alpha}\left(\operatorname{cl} \Omega, M_{r}(\mathbb{R})\right) \times C^{k, \alpha}\left(\operatorname{cl} \Omega, M_{r}(\mathbb{R})\right)$ to the space $C^{k, \alpha}\left(\operatorname{cl} \Omega, M_{r}(\mathbb{R})\right)$. 
(ii) The map $F \mapsto F^{-1}$ is analytic from $\left\{F \in C^{k, \alpha}\left(\operatorname{cl} \Omega, M_{r}(\mathbb{R})\right): \operatorname{det} F \neq 0\right.$ on $\left.\operatorname{cl} \Omega\right\}$ to itself, and its differential at the element $F_{0}$ is given by the map $M \vdash-F_{0}^{-1} \cdot M \cdot F_{0}^{-1}$.

We also mention that continuous (multi)linear operators between normed spaces are analytic (cf. e.g. Prodi and Ambrosetti [22]).

3. An analyticity result for multiple eigenvalues. As it is often done in the study of domain dependent problems, we transform our boundary value problem on $\phi(\Omega)$ into a problem on $\Omega$ by means of the following Proposition 3.1, whose validity can be easily verified by Lemma 2.3 and by the chain rule. Right after the statement of Proposition 3.1, we introduce some notation and we explain the structure of this section.

Proposition 3.1. Let $k \in \mathbb{N} \backslash\{0,1\}, \alpha \in] 0,1]$. Let $\Omega$ be an open bounded connected subset of $\mathbb{R}^{n}$ of class $C^{1}$. Let $\left.\phi \in C^{k, \alpha}\left(\mathrm{cl} \Omega, \mathbb{R}^{n}\right) \cap \mathcal{A}, \lambda \in\right] 0,+\infty[$. Then the function $v \in C^{k, \alpha}(\operatorname{cl} \phi(\Omega))$ satisfies the boundary value problem (2.6) if and only if the function $u \equiv v \circ \phi$ belongs to $C^{k, \alpha}(\mathrm{cl} \Omega)$ and satisfies the boundary value problem

$$
\begin{cases}-\Delta_{\phi} u=\lambda u & \text { in } \Omega \\ u=0 & \text { on } \partial \Omega\end{cases}
$$

where $\Delta_{\phi} u \equiv\left[\Delta\left(u \circ \phi^{(-1)}\right)\right] \circ \phi$. Furthermore

$$
\Delta_{\phi} u=\operatorname{tr}\left\{\left[(D \phi)^{-1}\right]^{t} \cdot(H u) \cdot\left[(D \phi)^{-1}\right]\right\}+\sum_{i, s, l=1}^{n} \frac{\partial u}{\partial x_{i}} \frac{\partial \sigma_{i s}}{\partial x_{l}} \sigma_{l s},
$$

where $\sigma_{i s}$ is the $(i, s)$ entry of the matrix $(D \phi)^{-1}$, and where $H u \equiv\left(\frac{\partial^{2} u}{\partial x_{i} \partial x_{s}}\right)_{i, s=1, \ldots, n}$. The operator $\Delta_{\phi}$ is uniformly elliptic in $\Omega$, and its ellipticity constant in $\Omega$ is greater or equal to the constant $\min _{x \in \mathrm{c} \Omega} \min _{\left\{\xi \in \mathbb{R}^{n}:|\xi|=1\right\}}\left|\left[(D \phi)^{-1}\right]^{t} \cdot \xi\right|^{2}$.

We now introduce some notation. For all $\phi \in C^{k, \alpha}\left(\operatorname{cl} \Omega, \mathbb{R}^{n}\right) \cap \mathcal{A}$, with $k, j \in \mathbb{N}$, $k \geq 2, j \geq 1, \alpha \in] 0,1\left[\right.$, we set $C_{0}^{k, \alpha}(\operatorname{cl} \Omega) \equiv\left\{u \in C^{k, \alpha}(\operatorname{cl} \Omega): u_{\mid \partial \Omega}=0\right\}$, and

$$
E_{\phi, j} \equiv\left\{u \in C_{0}^{k, \alpha}(\operatorname{cl} \Omega):-\Delta_{\phi} u=\lambda_{j}[\phi] u \text { in } \Omega\right\} .
$$

Thus the multiplicity of $\lambda_{j}[\phi]$ equals $\operatorname{dim} E_{\phi, j}$. Then we denote by $L_{\phi}^{2}(\Omega)$ the Hilbert space of the (equivalence classes) of functions of $\Omega$ to $\mathbb{R}$ that are square summable with respect to the measure $|\operatorname{det} D \phi| d x$, equipped with the scalar product defined by

$$
<u_{1}, u_{2}>_{\phi} \equiv \int_{\Omega} u_{1} u_{2}|\operatorname{det} D \phi| d x, \quad \forall u_{1}, u_{2} \in L_{\phi}^{2}(\Omega) .
$$

Then we set

$$
\mathcal{M}_{k, \alpha, j, m} \equiv\left\{\phi \in C^{k, \alpha}\left(\mathrm{cl} \Omega, \mathbb{R}^{n}\right) \cap \mathcal{A}: \operatorname{dim} E_{\phi, j}=m\right\}
$$


for all $m \in \mathbb{N} \backslash\{0\}$. Now we fix an element $\tilde{\phi} \in \mathcal{M}_{k, \alpha, j, m}$. For all $\phi \in \mathcal{M}_{k, \alpha, j, m}$, we denote by $P_{\phi, j}$ the orthogonal projection of $\left(L_{\tilde{\phi}}^{2}(\Omega),<\cdot, \cdot>_{\tilde{\phi}}\right)$ onto the eigenspace $E_{\phi}, j$.

Our goal is to prove that the functions which map $\phi \in \mathcal{M}_{k, \alpha, j, m}$ to $\lambda_{j}[\phi] \in \mathbb{R}$ and to $P_{\phi, j} \in \mathcal{L}\left(C_{0}^{k, \alpha}(\operatorname{cl} \Omega), C_{0}^{k, \alpha}(\operatorname{cl} \Omega)\right)$, are real analytic around $\tilde{\phi}$, in the sense that they admit a real analytic extension in a neighborhood of $\tilde{\phi}$. As a first step, we consider the restriction $p_{\phi, j}$ of $P_{\phi, j}$ to $E_{\tilde{\phi}, j}$, and we note that the triple $\left(\phi, p_{\phi, j}, \lambda_{j}[\phi]\right)$ is characterized by a certain system of equations (cf. Theorem 3.6), that we recast in the form of an abstract equation

$$
F[\phi, p, \lambda]=0 .
$$

In order to analyze equation (3.5), we analyze the corresponding operator $F$ in Theorem 3.13. Now it may be natural to think of applying the Implicit Function Theorem to equation (3.5) in order to deduce the regularity of the dependence of $(p, \lambda)$ as a function of $\phi$. However, this cannot be done, because the linearized $d_{(p, \lambda)} F$ at the reference state $\left(\tilde{\phi}, I, \lambda_{j}[\tilde{\phi}]\right)$ is not onto the target space of $F$ (cf. Theorem 3.13). We circumvent this difficulty by introducing the projection $\Pi$ onto the image of the linearized operator $d_{(p, \lambda)} F\left[\tilde{\phi}, I, \lambda_{j}[\tilde{\phi}]\right]$, and by considering the modified equation $\Pi \circ F[\phi, p, \lambda]=0$, which obviously contains the zeros of equation (3.5). Then we show that we can apply the Implicit Function Theorem to equation $\Pi \circ F[\phi, p, \lambda]=0$, and we obtain $(p, \lambda)$ as a certain functional of $\phi$ (cf. Theorem 3.34). In order to show that such implicitly defined functional coincides on $\mathcal{M}_{k, \alpha, j, m}$ with $\phi \rightarrow\left(p_{\phi, j}, \lambda_{j}[\phi]\right)$, a fact which we state in our main Theorem 3.34, we need to show that $p_{\phi, j}$ is continuous at $\tilde{\phi}$ for $\phi \in \mathcal{M}_{k, \alpha, j, m}$ (see Lemma 3.20 and Proposition 3.30) and, in order to deduce the regularity of the dependence of $P_{\phi, j}$ upon $\phi$ from the corresponding result for $p_{\phi, j}$, we shall prove the technical Lemma 3.32. Finally, in the last part of the section, we shall derive the Hadamard formulae for the derivatives of $\lambda_{j}[\cdot]$ along a curve of deformations of $\tilde{\phi}$ on $\mathcal{M}_{k, \alpha, j, m}$.

We now introduce a system of equations involving $\left(\phi, p_{\phi, j} \lambda_{j}[\phi]\right)$ by means of the following result.

Theorem 3.6. Let $k \in \mathbb{N} \backslash\{0,1\}, j, m \in \mathbb{N} \backslash\{0\}, \alpha \in] 0,1[$. Let $\Omega$ be a bounded open connected subset of $\mathbb{R}^{n}$ of class $C^{k, \alpha}$. Let $\tilde{\phi} \in \mathcal{M}_{k, \alpha, j, m}$. If $\phi \in \mathcal{M}_{k, \alpha, j, m}$, then the triple $\left(\phi, p_{\phi, j}, \lambda_{j}[\phi]\right)$, where $p_{\phi, j} \equiv P_{\phi, j \mid E_{\tilde{\phi}, j}}$ and where $P_{\phi, j}$ denotes the orthogonal projection of $L_{\tilde{\phi}}^{2}(\Omega)$ onto $E_{\phi, j}$, satisfies the system

$$
\begin{cases}\Delta_{\phi} \circ p_{\phi, j}+\lambda_{j}[\phi] p_{\phi, j}=0 & \text { in } \mathcal{L}\left(E_{\tilde{\phi}, j}, C^{k-2, \alpha}(\mathrm{cl} \Omega)\right), \\ <\left(I-p_{\phi, j}\right)(a), p_{\phi, j}(b)>_{\tilde{\phi}}=0, & \forall(a, b) \in E_{\tilde{\phi}, j}^{2},\end{cases}
$$

where I denotes the inclusion map of $E_{\tilde{\phi}, j}$ in $C_{0}^{k, \alpha}(\mathrm{cl} \Omega)$. Conversely, there exists an open neighborhood $\mathcal{V}$ of $\left(\tilde{\phi}, I, \lambda_{j}[\tilde{\phi}]\right)$ in $\left(C^{k, \alpha}\left(\operatorname{cl} \Omega, \mathbb{R}^{n}\right) \cap \mathcal{A}\right) \times \mathcal{L}\left(E_{\tilde{\phi}, j}, C_{0}^{k, \alpha}(\operatorname{cl} \Omega)\right) \times \mathbb{R}$ such that if $(\phi, p, \lambda) \in \mathcal{V}$ and if

$$
\begin{cases}\Delta_{\phi} \circ p+\lambda p=0 & \text { in } \mathcal{L}\left(E_{\tilde{\phi}, j}, C^{k-2, \alpha}(\mathrm{cl} \Omega)\right), \\ <(I-p)(a), p(b)>_{\tilde{\phi}}=0, & \forall(a, b) \in E_{\tilde{\phi}, j}^{2}\end{cases}
$$

then $p=P_{\phi,\left.\right|_{\mid} \tilde{\phi}_{\tilde{\phi}, j}}, \lambda=\lambda_{j}[\phi], \phi \in \mathcal{M}_{k, \alpha, j, m}$. 
Proof. The first part of the statement is obvious. We now prove the second part. Since $\lambda_{j}[\tilde{\phi}]$ has multiplicity $m$, we can assume that $\lambda_{j}[\tilde{\phi}]=\cdots=\lambda_{j+m-1}[\tilde{\phi}]$. By the continuous dependence of the eigenvalues upon $\phi$ (cf. Theorem 2.5), there exists an open interval $J$ containing $\lambda_{j}[\tilde{\phi}]$, and an open neighborhood $\mathcal{W}$ of $\tilde{\phi}$ such that $\lambda_{h}[\phi] \in J$ for $h=j, \ldots, j+m-1, \lambda_{h}[\phi] \notin J$ for $h \in \mathbb{N} \backslash\{0, j, \ldots, j+m-1\}$ and $\phi \in \mathcal{W}$. A simple contradiction argument shows that there exists an open neighborhood $\mathcal{J}$ of $I$ such that if $p \in \mathcal{J}$, then $p$ is injective. Accordingly $\operatorname{dim} p\left(E_{\tilde{\phi}, j}\right)=m$, for all $p \in \mathcal{J}$. Now, if $(\phi, p, \lambda) \in \mathcal{W} \times \mathcal{J} \times J \equiv \mathcal{V}$, and if equation (3.8) is satisfied, then $\lambda$ is an eigenvalue whose corresponding eigenspace contains $p\left(E_{\tilde{\phi}, j}\right)$, and thus $\lambda$ has multiplicity at least $m$. Since $\lambda \in J, \phi \in \mathcal{W}$, we must have $\lambda \in\left\{\lambda_{j}[\phi], \ldots, \lambda_{j+m-1}[\phi]\right\}$, and thus the multiplicity of $\lambda$ is at most $m$. Then the multiplicity is $m$, equality $\lambda=\lambda_{j}[\phi]=\ldots=\lambda_{j+m-1}[\phi]$ holds, and $p\left(E_{\tilde{\phi}, j}\right)=E_{\phi, j}$. We still have to prove that $p$ coincides with the restriction to $E_{\tilde{\phi}, j}$ of the orthogonal projection of $L_{\tilde{\phi}}^{2}(\Omega)$ onto $E_{\phi, j}$. Let $D \equiv E_{\tilde{\phi}, j} \cap E_{\phi, j}, E_{\tilde{\phi}, j} \equiv \tilde{Q} \oplus D$, with $\tilde{Q} \perp D$, and $E_{\phi, j}=D \oplus Q$, with $D \perp Q$. Let the space $H$ be such that $L_{\tilde{\phi}}^{2}(\Omega)=(\tilde{Q} \oplus D \oplus Q) \oplus H$, with $H \perp(\tilde{Q} \oplus D \oplus Q)$, where all direct sums have been taken in $L_{\tilde{\phi}}^{2}(\Omega)$. We set $P(\tilde{q}+d+q+h) \equiv p(\tilde{q}+d)+q$, for all $(\tilde{q}, d, q, h) \in \tilde{Q} \times D \times Q \times H$. Now, we show that $P$ is the orthogonal projection of $L_{\tilde{\phi}}^{2}(\Omega)$ onto $E_{\phi, j}$. It clearly suffices to verify that

$$
<(I-P)(\tilde{q}+d+q+h), P\left(\tilde{q}_{1}+d_{1}+q_{1}+h_{1}\right)>_{\tilde{\phi}}=0,
$$

for all $(\tilde{q}, d, q, h),\left(\tilde{q}_{1}, d_{1}, q_{1}, h_{1}\right) \in \tilde{Q} \times D \times Q \times H$. By the second equation of (3.8) and by the orthogonality of $h$ to $E_{\phi, j}$, the left hand side of (3.9) equals

$$
<\tilde{q}+d-p\left(\tilde{q}_{1}+d\right), q_{1}>_{\tilde{\phi}} .
$$

We note that $E_{\phi, j}=p\left(E_{\tilde{\phi}, j}\right)$ and accordingly, there exists $w \in E_{\tilde{\phi}, j}$ such that $q_{1}=p(w)$. Then the scalar product in (3.10) vanishes by the second equation of (3.8), and thus we have proved that condition (3.9) holds.

We now note that one can think of the set of the solutions of system (3.8) as the set of zeros of a certain function $F$. We introduce and analyze the function $F$ in Theorem 3.13. To do so however, we need the following technical statement, whose validity can be easily verified by Lemma 2.3 , by the chain rule, and by standard results of elliptic theory (cf. e.g. Nečas [20, Theorem 3.1, p. 135], Gilbarg and Trudinger [8, Theorem 6.19, p. 111 and Theorem 8.12, p. 186], Troianiello [23, Theorem 3.23, p. 189]).

Theorem 3.11. Let $k \in \mathbb{N} \backslash\{0,1\}, j, m \in \mathbb{N} \backslash\{0\}, \alpha \in] 0,1[$. Let $\Omega$ be a bounded open connected subset of $\mathbb{R}^{n}$ of class $C^{k, \alpha}$. Let $\tilde{\phi} \in \mathcal{M}_{k, \alpha, j, m}$. Then

$$
\left\{\Delta_{\tilde{\phi}} u+\lambda_{j}[\tilde{\phi}] u: u \in C_{0}^{k, \alpha}(\operatorname{cl} \Omega)\right\}=\left\{f \in C^{k-2, \alpha}(\operatorname{cl} \Omega):<f, w>_{\tilde{\phi}}=0, \forall w \in E_{\tilde{\phi}, j}\right\} .
$$

We are now ready to prove the following result.

Theorem 3.13. Let $k \in \mathbb{N} \backslash\{0,1\}, j, m \in \mathbb{N} \backslash\{0\}, \alpha \in] 0,1[$. Let $\Omega$ be a bounded open connected subset of $\mathbb{R}^{n}$ of class $C^{k, \alpha}$. Let $\tilde{\phi} \in \mathcal{M}_{k, \alpha, j, m}$. Let $F$ be the map of 
$\left(C^{k, \alpha}\left(\mathrm{cl} \Omega, \mathbb{R}^{n}\right) \cap \mathcal{A}\right) \times \mathcal{L}\left(E_{\tilde{\phi}, j}, C_{0}^{k, \alpha}(\mathrm{cl} \Omega)\right) \times \mathbb{R} \quad$ to $\quad \mathcal{L}\left(E_{\tilde{\phi}, j}, C^{k-2, \alpha}(\mathrm{cl} \Omega)\right) \times \mathcal{B}\left(E_{\tilde{\phi}, j}^{2}, \mathbb{R}\right)$ defined by

$$
F[\phi, p, \lambda] \equiv\left(\Delta_{\phi} \circ p+\lambda p,<(I-p)(\cdot), p(\cdot)>_{\tilde{\phi}}\right),
$$

for all $(\phi, p, \lambda) \in\left(C^{k, \alpha}\left(\mathrm{cl} \Omega, \mathbb{R}^{n}\right) \cap \mathcal{A}\right) \times \mathcal{L}\left(E_{\tilde{\phi}, j}, C_{0}^{k, \alpha}(\mathrm{cl} \Omega)\right) \times \mathbb{R}$, where I denotes the inclusion of $E_{\tilde{\phi}, j}$ in $C_{0}^{k, \alpha}(\mathrm{cl} \Omega)$. Then the map $F$ is real analytic. Let

$$
\begin{gathered}
\mathcal{Y} \equiv\left\{M \in \mathcal{L}\left(E_{\tilde{\phi}, j}, C^{k-2, \alpha}(\operatorname{cl} \Omega)\right): M\left(E_{\tilde{\phi}, j}\right) \text { is orthogonal to } E_{\tilde{\phi}, j} \text { in } L_{\tilde{\phi}}^{2}(\Omega)\right\}, \\
\mathcal{Z} \equiv\{\mu I: \mu \in \mathbb{R}\} .
\end{gathered}
$$

Then $\mathcal{Y} \cap \mathcal{Z}=\{0\}$ and the space $(\mathcal{Y} \oplus \mathcal{Z}) \times \mathcal{B}\left(E_{\tilde{\phi}, j}^{2}, \mathbb{R}\right)$ is a closed subspace of $\mathcal{L}\left(E_{\tilde{\phi}, j}, C^{k-2, \alpha}(\mathrm{cl} \Omega)\right) \times \mathcal{B}\left(E_{\tilde{\phi}, j}^{2}, \mathbb{R}\right)$. The differential $d_{(p, \lambda)} F\left[\tilde{\phi}, I, \lambda_{j}[\tilde{\phi}]\right]$ is a linear homeomorphism of $\mathcal{L}\left(E_{\tilde{\phi}, j}, C_{0}^{k, \alpha}(\mathrm{cl} \Omega)\right) \times \mathbb{R}$ onto $(\mathcal{Y} \oplus \mathcal{Z}) \times \mathcal{B}\left(E_{\tilde{\phi}, j}^{2}, \mathbb{R}\right)$.

Let $\left\{\tilde{u}_{1}, \ldots, \tilde{u}_{m}\right\}$ be an orthonormal basis for $E_{\tilde{\phi}, j}$ with respect to the scalar product of $L_{\tilde{\phi}}^{2}(\Omega)$. Let $\Pi_{1}$ be the map of $\mathcal{L}\left(E_{\tilde{\phi}, j}, C^{k-2, \alpha}(\operatorname{cl} \Omega)\right)$ to $\mathcal{Y} \oplus \mathcal{Z}$ defined by

$$
\Pi_{1}(M) \equiv M[\cdot]-\sum_{l=1}^{m}<M[\cdot], \tilde{u}_{l}>_{\tilde{\phi}} \tilde{u}_{l}+<M\left[\tilde{u}_{1}\right], \tilde{u}_{1}>_{\tilde{\phi}} I,
$$

where I is the inclusion of $E_{\tilde{\phi}, j}$ into $C^{k-2, \alpha}(\mathrm{cl} \Omega)$. The map $\Pi_{1}$ is linear and continuous, and restricts the identity on $\mathcal{Y} \oplus \mathcal{Z}$.

Proof. To shorten our notation, we set $\tilde{\lambda} \equiv \lambda_{j}[\tilde{\phi}], \quad \mathcal{X}_{1} \equiv \mathcal{L}\left(E_{\tilde{\phi}, j}, C_{0}^{k, \alpha}(\operatorname{cl} \Omega)\right)$, $\mathcal{X}_{2} \equiv \mathcal{L}\left(E_{\tilde{\phi}, j}, C^{k-2, \alpha}(\mathrm{cl} \Omega)\right), \mathcal{B} \equiv \mathcal{B}\left(E_{\tilde{\phi},}^{2}, \mathbb{R}\right)$. The real analyticity of $F$ is an immediate consequence of (3.3) and of Lemma 2.7. Equality $\mathcal{Y} \cap \mathcal{Z}=\{0\}$ is obvious. By simple computations, it can be easily verified that $\Pi_{1}$ is linear and continuous and that $\Pi_{1 \mid \mathcal{Y} \oplus \mathcal{Z}}$ restricts the identity on $\mathcal{Y} \oplus \mathcal{Z}$. Then $\mathcal{Y} \oplus \mathcal{Z}=\operatorname{Ker}\left(I-\Pi_{1}\right)$ and, accordingly, $\mathcal{Y} \oplus \mathcal{Z}$ is closed. By standard calculus and by (3.14), we have

$$
d_{(p, \lambda)} F[\tilde{\phi}, I, \tilde{\lambda}](Q, \mu)=\left(\Delta_{\tilde{\phi}} \circ Q+\tilde{\lambda} Q+\mu I, B[Q]\right), \forall(Q, \mu) \in \chi_{1} \times \mathbb{R},
$$

where $B[Q]$ is the element of $\mathcal{B}$ defined by $B[Q](a, b) \equiv-<Q(a), b>_{\tilde{\phi}}$, for all $a, b \in E_{\tilde{\phi}, j}$. We now prove that $d_{(p, \lambda)} F[\tilde{\phi}, I, \tilde{\lambda}]$ is injective. Assume that there exist $Q \in \mathcal{X}_{1}, \mu \in \mathbb{R}$ such that

$$
\begin{cases}\Delta_{\tilde{\phi}} \circ Q+\tilde{\lambda} Q+\mu I=0 & \text { in } \mathcal{X}_{2}, \\ -<Q(a), b>_{\tilde{\phi}}=0, & \forall a, b \in E_{\tilde{\phi}, j} .\end{cases}
$$

Then we have

$$
\Delta_{\tilde{\phi}}(Q(u))+\tilde{\lambda}(Q(u))+\mu u=0, \quad \text { in } \Omega,
$$

for all $u \in E_{\tilde{\phi}, j}$, and thus, by Theorem 3.11, we obtain $\mu=0$. Then, by equation (3.17), we conclude that $Q(u) \in E_{\tilde{\phi}, j}$, for all $u \in E_{\tilde{\phi}, j}$, and thus, by the second 
equation of (3.16), we obtain $\left\langle Q(u), Q(u)>_{\tilde{\phi}}=0\right.$, for all $u \in E_{\tilde{\phi}, j}$. Accordingly $Q=0$, and thus $d_{(p, \lambda)} F[\tilde{\phi}, I, \tilde{\lambda}]$ is injective. We now show that $d_{(p, \lambda)} F[\tilde{\phi}, \tilde{I}, \tilde{\lambda}]\left(\mathcal{X}_{1} \times \mathbb{R}\right)=$ $(\mathcal{Y} \oplus \mathcal{Z}) \times \mathcal{B}$. If the pair $(M, S)$ belongs to $d_{(p, \lambda)} F[\tilde{\phi}, I, \tilde{\lambda}]\left(\mathcal{X}_{1} \times \mathbb{R}\right)$, then the system

$$
\begin{cases}\Delta_{\tilde{\phi}} \circ Q+\tilde{\lambda} Q+\mu I=M & \text { in } \mathcal{X}_{2}, \\ -<Q(a), b>_{\tilde{\phi}}=S(a, b), & \forall a, b \in E_{\tilde{\phi}, j},\end{cases}
$$

has at least one solution $(Q, \mu) \in \mathcal{X}_{1} \times \mathbb{R}$. Then we have $\Delta_{\tilde{\phi}}(Q(v))+\tilde{\lambda} Q(v)=$ $M[v]-\mu v$, for all $v \in E_{\tilde{\phi}, j}$ and thus, by Theorem 3.11, we obtain

$$
<M\left[\tilde{u}_{i}\right], \tilde{u}_{s}>_{\tilde{\phi}}=\mu<\tilde{u}_{i}, \tilde{u}_{s}>_{\tilde{\phi}}, \quad \forall i, s \in\{1, \ldots, m\} .
$$

Now, we can write $M\left[\tilde{u}_{i}\right]$ as $M\left[\tilde{u}_{i}\right]=\sum_{l=1}^{m} \theta_{l i} \tilde{u}_{l}+v_{i}$, with $\theta_{l i} \in \mathbb{R}, v_{i} \in C^{k-2, \alpha}(\mathrm{cl} \Omega)$ and $<v_{i}, \tilde{u}_{s}>_{\tilde{\phi}}=0$, for all $s=1, \ldots, m$. By (3.19) and by our choice of $\tilde{u}_{1}, \ldots, \tilde{u}_{m}$, we have $\theta_{l i}=\mu<\tilde{u}_{l}, \tilde{u}_{i}>_{\tilde{\phi}}$. Then we have $M \in \mathcal{Y} \oplus \mathcal{Z}$. Conversely, let $(M, S) \in$ $(\mathcal{Y} \oplus \mathcal{Z}) \times \mathcal{B}$. Then $M=M_{1}+M_{2}$, with $M_{1} \in \mathcal{Y}, M_{2} \in \mathcal{Z}$. In particular, there exists $\mu \in \mathbb{R}$ such that $M_{2}=\mu I$, and by our choice of $\tilde{u}_{1}, \ldots, \tilde{u}_{m}$, and by the orthogonality of the image of $M_{1}$ to $E_{\tilde{\phi}, j}$, we obtain (3.19) and thus by Theorem 3.11, there exist $\tilde{\omega}_{i} \in C_{0}^{k, \alpha}(\operatorname{cl} \Omega)$ such that $\Delta_{\tilde{\phi}} \tilde{\omega}_{i}+\tilde{\lambda} \tilde{\omega}_{i}=M\left[\tilde{u}_{i}\right]-\mu \tilde{u}_{i}$ in $\Omega$, for all $i=1, \ldots, m$. Now we set $\omega_{i} \equiv \tilde{\omega}_{i}+\sum_{l=1}^{m} a_{i l} \tilde{u}_{l}$, with $a_{i l} \in \mathbb{R}$, for all $i, l \in\{1, \ldots, m\}$, and we determine the coefficients $a_{i l}$ so that $-<\omega_{i}, \tilde{u}_{s}>_{\tilde{\phi}}=S\left(\tilde{u}_{i}, \tilde{u}_{s}\right)$, for all $i, s \in\{1, \ldots, m\}$. Clearly, we can take $a_{i l}=-S\left(\tilde{u}_{i}, \tilde{u}_{l}\right)-<\tilde{\omega}_{i}, \tilde{u}_{l}>_{\tilde{\phi}}$. Now, let $Q$ be the element of $\mathcal{X}_{1}$ defined by $Q\left[\tilde{u}_{i}\right]=\omega_{i}$. Then we must necessarily have $d_{(p, \lambda)} F[\tilde{\phi}, I, \tilde{\lambda}](Q, \mu)=(M, S)$.

We now prove the continuity of the dependence of $P_{\phi, j}$ at $\phi=\tilde{\phi}$, for $\phi \in \mathcal{M}_{k, \alpha, j, m}$ by means of the following two statements.

Lemma 3.20. Let $k \in \mathbb{N} \backslash\{0,1\}, j, m \in \mathbb{N} \backslash\{0\}, \alpha \in] 0,1[$. Let $\Omega$ be a bounded open connected subset of $\mathbb{R}^{n}$ of class $C^{k, \alpha}$. Let $\left\{\phi_{l}\right\}_{l \in \mathbb{N}}$ be a sequence in $\mathcal{M}_{k, \alpha, j, m}$ that converges to $\tilde{\phi} \in \mathcal{M}_{k, \alpha, j, m}$ in $C^{k, \alpha}\left(\mathrm{cl} \Omega, \mathbb{R}^{n}\right)$. Let $u_{l} \in E_{\phi_{l}, j},\left\langle u_{l}, u_{l}>_{\tilde{\phi}}=1\right.$, for all $l \in \mathbb{N}$. Then there exists a subsequence $\left\{u_{l_{r}}\right\}_{r \in \mathbb{N}}$ of $\left\{u_{l}\right\}_{l \in \mathbb{N}}$ and $u \in E_{\tilde{\phi}, j}$ such that $\lim _{r \rightarrow+\infty} u_{l_{r}}=u$ in $C^{k, \alpha}(\mathrm{cl} \Omega)$, and $<u, u>_{\tilde{\phi}}=1$.

Proof. Since the map of $C^{k, \alpha}\left(\mathrm{cl} \Omega, \mathbb{R}^{n}\right) \cap \mathcal{A}$ to $C^{k-1, \alpha}\left(\mathrm{cl} \Omega, M_{n}(\mathbb{R})\right)$ that takes $\phi$ to $(D \phi)^{-1}$ is real analytic, the same map is also bounded around $\tilde{\phi}$. Accordingly, there exist a neighborhood $\mathcal{W}$ of $\tilde{\phi}$ and a constant $c>0$ such that

$$
\begin{aligned}
& \frac{1}{c}<\min _{x \in \mathrm{cl} \Omega} \min _{\left\{\xi \in \mathbb{R}^{n}:|\xi|=1\right\}}\left|\left[(D \phi)^{-1}\right]^{t} \cdot \xi\right|^{2}, \\
& \sup _{i, s=1, \ldots, n}\left\|\left[(D \phi)^{-1}\right]_{i s}\right\|_{C^{k-1, \alpha}(\mathrm{cl} \Omega)}<c, \\
& \sup _{i, s, l=1, \ldots, n}\left\|\frac{\partial\left[(D \phi)^{-1}\right]_{i s}}{\partial x_{l}}\left[(D \phi)^{-1}\right]_{l s}\right\|_{C^{k-2, \alpha}(\mathrm{cl} \Omega)}<c,
\end{aligned}
$$

for all $\phi \in \mathcal{W}$. By standard elliptic estimates (cf. e.g. Troianiello [23, Theorem 3.28 (ii), p. 194]), there exists a constant $c_{1}>0$ depending only on $c, n, \Omega, k, \alpha, \mathcal{W}$ such that 


$$
\|u\|_{C^{k, \alpha}(\mathrm{cl} \Omega)} \leq c_{1}\left\|\Delta_{\phi} u\right\|_{C^{k-2, \alpha}(\mathrm{cl} \Omega)}, \quad \forall u \in C_{0}^{k, \alpha}(\mathrm{cl} \Omega), \quad \forall \phi \in \mathcal{W} .
$$

Since $\lim _{l \rightarrow \infty} \phi_{l}=\tilde{\phi}$, then by possibly neglecting a finite number of terms of the sequence, we can assume that

$$
\left\|u_{l}\right\|_{C^{k, \alpha}(\mathrm{cl} \Omega)} \leq c_{1} \lambda_{j}\left[\phi_{l}\right]\left\|u_{l}\right\|_{C^{k-2, \alpha}(\mathrm{cl} \Omega)} \quad \forall l \in \mathbb{N}
$$

By Theorem 2.5, the function $\lambda_{j}[\cdot]$ is continuous. Then there exists $c_{2}>0$ such that

$$
c_{2} \equiv \sup _{l \in \mathbb{N}} \lambda_{j}\left[\phi_{l}\right]<+\infty
$$

By Lemma 2.1, the space $C^{k, \alpha}(\mathrm{cl} \Omega)$ is compactly imbedded in $C^{k-2, \alpha}(\mathrm{cl} \Omega)$, and $C^{k-2, \alpha}(\mathrm{cl} \Omega)$ is imbedded in $L^{2}(\Omega)$. Thus by Lion's Lemma (cf. for example Berger [1, p. 35]), there exists a constant $c_{3}>0$, depending only on $\Omega, n, k, \alpha, c_{1}, c_{2}$, such that

$$
\|u\|_{C^{k-2, \alpha}(\mathrm{cl} \Omega)} \leq \frac{1}{2 c_{1} c_{2}}\|u\|_{C^{k, \alpha}(\mathrm{cl} \Omega)}+c_{3}\|u\|_{L^{2}(\Omega)}, \quad \forall u \in C^{k, \alpha}(\operatorname{cl} \Omega) .
$$

Then by (3.23), (3.24), (3.25), we conclude that $\frac{1}{2}\left\|u_{l}\right\|_{C^{k, \alpha}(\mathrm{cl} \Omega)} \leq c_{1} c_{2} c_{3}\left\|u_{l}\right\|_{L^{2}(\Omega)}$, for all $l \in \mathbb{N}$. Since $|\operatorname{det} D \tilde{\phi}|$ is continuous and positive in $\mathrm{cl} \Omega$, we can assume that there exists a constant $c_{4}>0$ such that $0<c_{4}<\min _{x \in \operatorname{cl} \Omega}|\operatorname{det} D \tilde{\phi}|$. Since $\int_{\Omega} u_{l}^{2}|\operatorname{det} D \tilde{\phi}| d x=1$, we conclude that

$$
\left\|u_{l}\right\|_{C^{k, \alpha}(\mathrm{cl} \Omega)} \leq 2 c_{1} c_{2} c_{3} c_{4}^{-1 / 2}, \quad \forall l \in \mathbb{N} .
$$

Now let $\beta \in] 0, \alpha\left[\right.$. By Lemma 2.1 (iv) there exists a subsequence $\left\{u_{l_{r}}\right\}_{r \in \mathbb{N}}$ of $\left\{u_{l}\right\}_{l_{\ell} \mathbb{N}}$, and $u \in C^{k, \alpha}(\mathrm{cl} \Omega)$ such that $\lim _{r \rightarrow+\infty} u_{l_{r}}=u$ in $C^{k, \beta}(\mathrm{cl} \Omega)$. Since $\lim _{r \rightarrow+\infty} \phi_{l_{r}}=\tilde{\phi}$ in $C^{k, \alpha}(\mathrm{cl} \Omega)$ and $\lim _{r \rightarrow+\infty} \lambda_{j}\left[\phi_{l_{r}}\right]=\lambda_{j}[\tilde{\phi}]$ (cf. Theorem 2.5), we have $\Delta_{\tilde{\phi}} u+\lambda_{j}[\tilde{\phi}] u=0$ in $\Omega$. Thus $u \in E_{\tilde{\phi}, j}$. Now, by (3.22), and by the membership of $u_{l_{r}} \in E_{\phi_{l_{r}}}, j$, and of $u \in E_{\tilde{\phi}, j}$, we have

$$
\left\|u_{l_{r}}-u\right\|_{C^{k, \alpha}(\mathrm{cl} \Omega)} \leq c_{1}\left\{\left\|\Delta_{\tilde{\phi}} u_{l_{r}}-\Delta_{\phi_{l_{r}}} u_{l_{r}}\right\|_{C^{k-2, \alpha}(\mathrm{cl} \Omega)}+\left\|\lambda_{j}\left[\phi_{l_{r}}\right] u_{l_{r}}-\lambda_{j}[\tilde{\phi}] u\right\|_{C^{k-2, \alpha}(\mathrm{cl} \Omega)}\right\},
$$

for all $r \in \mathbb{N}$. Since $C^{k, \beta}(\mathrm{cl} \Omega)$ is continuously imbedded in $C^{k-2, \alpha}(\mathrm{cl} \Omega)$, we have $\lim _{r \rightarrow+\infty} u_{l_{r}}=u$ in $C^{k-2, \alpha}(\operatorname{cl} \Omega)$. By Theorem 2.5, we have $\lim _{r \rightarrow+\infty} \lambda_{j}\left[\phi_{l_{r}}\right]=\lambda_{j}[\tilde{\phi}]$. Then we conclude that

$$
\lim _{r \rightarrow+\infty}\left\|\lambda_{j}\left[\phi_{l_{r}}\right] u_{l_{r}}-\lambda_{j}[\tilde{\phi}] u\right\|_{C^{k-2, \alpha}(\mathrm{cl} \Omega)}=0
$$

By inequality (3.26), the sequence $\left\{u_{l_{r}}\right\}_{r \in \mathbb{N}}$ is bounded in $C^{k, \alpha}(\mathrm{cl} \Omega)$. Then, by (3.3), the triangle inequality, the continuity of the pointwise product in $C^{k, \alpha}(\mathrm{cl} \Omega)$, and the convergence of $\left\{\phi_{l_{r}}\right\}_{r \in \mathbb{N}}$ to $\tilde{\phi}$ in $C^{k, \alpha}\left(\mathrm{cl} \Omega, \mathbb{R}^{n}\right)$, we conclude that

$$
\lim _{r \rightarrow+\infty}\left\|\Delta_{\tilde{\phi}} u_{l_{r}}-\Delta_{\phi_{l_{r}}} u_{l_{r}}\right\|_{C^{k-2, \alpha}(\mathrm{cl} \Omega)}=0 .
$$

By combining (3.27), (3.28), (3.29), we deduce that $\lim _{r \rightarrow+\infty} u_{l_{r}}=u$ in $C^{k, \alpha}(\mathrm{cl} \Omega)$. 
Proposition 3.30. Let $k \in \mathbb{N} \backslash\{0,1\}, j, m \in \mathbb{N} \backslash\{0\}, \alpha \in] 0,1[$. Let $\Omega$ be a bounded open connected subset of $\mathbb{R}^{n}$ of class $C^{k, \alpha}$. Let $\tilde{\phi} \in \mathcal{M}_{k, \alpha, j, m}$. Let $P_{\phi, j}$ be the restriction to $C_{0}^{k, \alpha}(\mathrm{cl} \Omega)$ of the orthogonal projection in $L_{\tilde{\phi}}^{2}(\Omega)$ of $L_{\tilde{\phi}}^{2}(\Omega)$ onto $E_{\phi, j}$, for all $\phi \in \mathcal{M}_{k, \alpha, j, m}$. Then the map of $\mathcal{M}_{k, \alpha, j, m}$ to $\mathcal{L}\left(C_{0}^{k, \alpha}(\mathrm{cl} \Omega), C_{0}^{k, \alpha}(\mathrm{cl} \Omega)\right)$ that takes $\phi$ to $P_{\phi, j}$ is continuous at $\tilde{\phi}$.

Proof. For all $\phi \in \mathcal{M}_{k, \alpha, j, m}$, we fix an orthonormal basis $\left\{u_{\phi, 1}, \ldots, u_{\phi, m}\right\}$ of $E_{\phi, j}$ in the scalar product of $L_{\tilde{\phi}}^{2}(\Omega)$. Clearly,

$$
P_{\phi, j}[u]=\sum_{s=1}^{m}<u, u_{\phi, s}>_{\tilde{\phi}} u_{\phi, s}, \quad \forall u \in C_{0}^{k, \alpha}(\operatorname{cl} \Omega) .
$$

Then $P_{\phi, j} \in \mathcal{L}\left(C_{0}^{k, \alpha}(\mathrm{cl} \Omega), C_{0}^{k, \alpha}(\mathrm{cl} \Omega)\right)$. To prove the continuity of the map which takes $\phi$ to $P_{\phi, j}$, it suffices to show that if a sequence $\left\{\phi_{l}\right\}_{l \in \mathbb{N}}$ converges to $\tilde{\phi}$ in $\mathcal{M}_{k, \alpha, j, m}$, then there exists a subsequence $\left\{\phi_{l_{r}}\right\}_{r \in \mathbb{N}}$ of $\left\{\phi_{l}\right\}_{l \in \mathbb{N}}$ such that $\left\{P_{\phi_{l_{r}}, j}\right\}_{r \in \mathbb{N}}$ converges to $P_{\tilde{\phi}, j}$ in $\mathcal{L}\left(C_{0}^{k, \alpha}(\mathrm{cl} \Omega), C_{0}^{k, \alpha}(\mathrm{cl} \Omega)\right)$. By the previous Lemma, there exist a subsequence $\left\{\phi_{l_{r}}\right\}_{r \in \mathbb{N}}$, and $\tilde{u}_{1}, \ldots, \tilde{u}_{m} \in E_{\tilde{\phi}, j}$ such that $\lim _{r \rightarrow+\infty} u_{\phi_{l r}, s}=\tilde{u}_{s}$, for all $s \in\{1, \ldots, m\}$. Clearly $\left\{\tilde{u}_{1}, \ldots, \tilde{u}_{m}\right\}$ is an orthonormal basis of $E_{\tilde{\phi}, j}$ and $P_{\tilde{\phi}, j}[u]=\sum_{s=1}^{m}<u, \tilde{u}_{s}>_{\tilde{\phi}} \tilde{u}_{s}$, for all $u \in C_{0}^{k, \alpha}(\mathrm{cl} \Omega)$. Then by the equality (3.31) and by the limiting relation $\lim _{r \rightarrow+\infty} u_{\phi l_{r}, s}=\tilde{u}_{s}$, we can easily deduce that $\lim _{r \rightarrow+\infty} P_{\phi_{l r}, j}=P_{\tilde{\phi}, j} \quad$ in $\mathcal{L}\left(C_{0}^{k, \alpha}(\mathrm{cl} \Omega), C_{0}^{k, \alpha}(\mathrm{cl} \Omega)\right)$.

We now prove the following technical statement, which we employ in the proof of our main Theorem 3.34 in order to deduce the regularity of the dependence of $P_{\phi, j}$ upon $\phi$ by the corresponding regularity result for $p_{\phi, j}$ upon $\phi$.

Lemma 3.32. Let $m, k \in \mathbb{N}, \alpha \in] 0,1\left[\right.$. Let $\Omega$ be a bounded open subset of $\mathbb{R}^{n}$. Let $\tau$ be a continuous function of $\mathrm{cl} \Omega$ to $] 0,+\infty\left[\right.$. Let $E$ be a subspace of $C_{0}^{k, \alpha}(\mathrm{cl} \Omega)$ of dimension $m$. Let I be the inclusion map of E into $C_{0}^{k, \alpha}(\mathrm{cl} \Omega)$. Then there exists an open neighborhood $\mathcal{U}$ of $I$ in the space $\mathcal{L}\left(E, C_{0}^{k, \alpha}(\mathrm{cl} \Omega)\right)$, and a real analytic map $\Gamma_{\tau}$ of $\mathcal{U}$ to $\mathcal{L}\left(C_{0}^{k, \alpha}(\mathrm{cl} \Omega), C_{0}^{k, \alpha}(\mathrm{cl} \Omega)\right)$, which takes an operator $T$ into the orthogonal projection $\Gamma_{\tau}[T]$ of $C_{0}^{k, \alpha}(\mathrm{cl} \Omega)$ onto $T(E)$ with respect to the scalar product defined by

$$
<u, v>\equiv \int_{\Omega} u v \tau d x, \quad \forall u, v \in C_{0}^{k, \alpha}(\operatorname{cl} \Omega)
$$

Proof. We can clearly assume that $m>0$. A simple contradiction argument shows that there exists an open neighborhood $\mathcal{U}$ of $I$ such that $T$ is injective, for all $T \in \mathcal{U}$. Since $E$ has finite dimension $m$, the dimension of $T(E)$ is exactly $m$, for all $T \in \mathcal{U}$. Let $E$ be generated by $\left\{u_{1}, \ldots, u_{m}\right\}$. Clearly, for all $T \in \mathcal{U}$, the set $\left\{T\left(u_{1}\right), \ldots\right.$, $\left.T\left(u_{m}\right)\right\}$ is a basis for $T(E)$, and the set of vectors $\left\{v_{1}[T], \ldots, v_{m}[T]\right\}$ defined by

$$
\begin{aligned}
v_{1}[T] & \equiv \frac{T\left(u_{1}\right)}{\left\{\int_{\Omega}\left[T\left(u_{1}\right)\right]^{2} \tau d x\right\}^{1 / 2}}, \\
v_{s+1}[T] & \equiv \frac{T\left(u_{s+1}\right)-\sum_{l=1}^{s} \int_{\Omega} T\left(u_{s+1}\right) v_{l}[T] \tau d x v_{l}[T]}{\left\{\int_{\Omega}\left[T\left(u_{s+1}\right)-\sum_{l=1}^{s} \int_{\Omega} T\left(u_{s+1}\right) v_{l}[T] \tau d x v_{l}[T]\right]^{2} \tau d x\right\}^{1 / 2}},
\end{aligned}
$$


for all $s=1, \ldots, m-1$, is an orthonormal basis for $T(E)$, with respect to the scalar product of (3.33). Since $\Gamma_{\tau}[T](u)=\sum_{l=1}^{m} \int_{\Omega} u v_{l}[T] \tau d x v_{l}[T]$, and the functions $v_{s}[\cdot]$, with $s=1, \ldots, m$ are real analytic, we conclude that $\Gamma_{\tau}[\cdot]$ is real analytic.

We are now ready to prove our main result.

Theorem 3.34. Let $k \in \mathbb{N} \backslash\{0,1\}, j, m \in \mathbb{N} \backslash\{0\}, \alpha \in] 0,1[$. Let $\Omega$ be a bounded open connected subset of $\mathbb{R}^{n}$ of class $C^{k, \alpha}$. Let $\tilde{\phi} \in \mathcal{M}_{k, \alpha, j, m}$. Let $\Pi_{1}$ be the map of (3.15). Let $\Pi$ be the map of $\mathcal{L}\left(E_{\tilde{\phi}, j}, C^{k-2, \alpha}(\mathrm{cl} \Omega)\right) \times \mathcal{B}\left(E_{\tilde{\phi}, j}^{2}, \mathbb{R}\right)$ to $(\mathcal{Y} \oplus \mathcal{Z}) \times \mathcal{B}\left(E_{\tilde{\phi}, j}^{2}, \mathbb{R}\right)$ defined by $\Pi(M, S) \equiv\left(\Pi_{1}(M), S\right)$, for all $(M, S) \in \mathcal{L}\left(E_{\tilde{\phi}, j}, C^{k-2, \alpha}(\mathrm{cl} \Omega)\right) \times \mathcal{B}\left(E_{\tilde{\phi}, j}^{2}, \mathbb{R}\right)$. Let $F$ be the map of Theorem 3.13. Let $P_{\phi, j}$ be the restriction to $C_{0}^{k, \alpha}(\mathrm{cl} \Omega)$ of the orthogonal projection of $L_{\tilde{\phi}}^{2}(\Omega)$ onto $E_{\phi, j}$, for all $\phi \in \mathcal{M}_{k, \alpha, j, m}$. Let $p_{\phi, j}$ be the restriction of $P_{\phi, j}$ to $E_{\tilde{\phi}, j}$. Then there exist an open neighborhood $\mathcal{W}$ of $\tilde{\phi}$ in $C^{k, \alpha}\left(\operatorname{cl} \Omega, \mathbb{R}^{n}\right) \cap \mathcal{A}$, an open neighborhood $\mathcal{U}$ of $I$ in $\mathcal{L}\left(E_{\tilde{\phi}, j}, C_{0}^{k, \alpha}(\mathrm{cl} \Omega)\right)$, an open interval $J$ of $\mathbb{R}$ containing $\lambda_{j}[\tilde{\phi}]$, a real analytic map $\hat{\lambda}_{\tilde{\phi}, j}$ of $\mathcal{W}$ to $J$, a real analytic map $\hat{p}_{\tilde{\phi}, j}$ of $\mathcal{W}$ to $\mathcal{U}$, a real analytic map $\hat{P}_{\tilde{\phi}, j}$ of $\mathcal{W}$ to $\mathcal{L}\left(C_{0}^{k, \alpha}(\mathrm{cl} \Omega), C_{0}^{k, \alpha}(\mathrm{cl} \Omega)\right)$ such that $\hat{P}_{\tilde{\phi}, j}[\phi]$ is the orthogonal projection of $C_{0}^{k, \alpha}(\mathrm{cl} \Omega)$ onto the space $\hat{p}_{\tilde{\phi}, j}[\phi]\left(E_{\tilde{\phi}, j}\right)$ with respect to the product of $L_{\tilde{\phi}}^{2}(\Omega)$ for all $\phi \in \mathcal{W}$, and such that

$$
\begin{gathered}
\{(\phi, p, \lambda) \in \mathcal{W} \times \mathcal{U} \times J: \Pi(F[\phi, p, \lambda])=0\}=\left\{\left(\phi, \hat{p}_{\tilde{\phi}, j}[\phi], \hat{\lambda}_{\tilde{\phi}, j}[\phi]\right): \phi \in \mathcal{W}\right\}, \\
\hat{\lambda}_{\tilde{\phi}, j}[\phi]=\lambda_{j}[\phi], \quad \forall \phi \in \mathcal{W} \cap \mathcal{M}_{k, \alpha, j, m}, \\
\hat{p}_{\tilde{\phi}, j}[\phi]=p_{\phi, j}, \quad \forall \phi \in \mathcal{W} \cap \mathcal{M}_{k, \alpha, j, m}, \\
\hat{P}_{\tilde{\phi}, j}[\phi]=P_{\phi, j}, \quad \forall \phi \in \mathcal{W} \cap \mathcal{M}_{k, \alpha, j, m}, \\
\operatorname{dim} \hat{p}_{\tilde{\phi}, j}[\phi]\left(E_{\tilde{\phi}, j}\right)=\operatorname{dim} \hat{P}_{\tilde{\phi}, j}[\phi]\left(C_{0}^{k, \alpha}(\operatorname{cl} \Omega)\right)=m, \quad \forall \phi \in \mathcal{W} .
\end{gathered}
$$

Proof. By Theorem 3.13, we can apply the Implicit Function Theorem to equation $\Pi \circ F=0$, around the point $\left(\tilde{\phi}, I, \lambda_{j}[\tilde{\phi}]\right)$, and deduce the existence of $\mathcal{W}, \mathcal{U}, J$, $\hat{\lambda}_{\tilde{\phi}, j}, \hat{p}_{\tilde{\phi}, j}$, as in (3.35). By possibly shrinking $\mathcal{W}$ and $\mathcal{U}$, we can assume that $\mathcal{U}$ is contained in the neighborhood $\mathcal{U}$ of Lemma 3.32, with $\tau=|\operatorname{det} D \tilde{\phi}|$. Then, by setting $\hat{P}_{\tilde{\phi}, j}[\phi] \equiv \Gamma_{|\operatorname{det} D \tilde{\phi}|}\left[\hat{p}_{\phi, j}[\phi]\right]$ for all $\phi \in \mathcal{W}$ and, by invoking Theorem 2.5, Proposition 3.30 and Theorem 3.6, one can easily see that (3.36)-(3.39) hold.

REMARK 3.40. Although (3.36)-(3.39) hold, we do not expect, in general, that for $\phi \in \mathcal{W} \backslash \mathcal{M}_{k, \alpha, j, m}$ the value $\hat{\lambda}_{\tilde{\phi}, j}[\phi]$ of the function $\hat{\lambda}_{\tilde{\phi}_{j}[}[\cdot]$ coincides with $\lambda_{j}[\phi]$, and that the space $\hat{p}_{\tilde{\phi}, j}[\phi]\left(E_{\tilde{\phi}, j}\right)$ is the eigenspace $E_{\phi, j}$.

REMARK 3.41. If $m=1, \tilde{\phi} \in \mathcal{M}_{k, \alpha, j, 1}$ then, by the continuity of $\lambda_{j}[\cdot]$ around $\tilde{\phi}$, one easily deduces that $\tilde{\phi}$ is interior to $\mathcal{M}_{k, \alpha, j, 1}$. By the previous Theorem, $\lambda_{j}[\phi]$ and $P_{\phi, j}$ are real analytic in $\phi$, for $\phi$ in a neighborhood of $\tilde{\phi}$. In particular, a suitably normalized eigenvector generating $E_{\phi, j}$ depends real analytically upon $\phi$ in a neighborhood of $\tilde{\phi}$. Such an analyticity result is well known (cf. Henry $[9, \S 3.2]$.)

We now show that Theorem 3.34 allows us, in particular, to prove the Hadamard formulae for the derivatives of the function $\lambda_{j}[\cdot]$ along a curve $\gamma(\cdot)$ of deformations of $\tilde{\boldsymbol{\phi}}$ on $\mathcal{M}_{k, \alpha, j, m}$. (See also Henry $[\mathbf{9}, \S 4.1]$ and Desaint and Zolésio [6].) To do 
so, we introduce the following technical Lemma, whose proof exploits a computation of Micheletti [18, pp. 161-163].

Lemma 3.42. Let $k \in \mathbb{N} \backslash\{0,1\}, j, m \in \mathbb{N} \backslash\{0\}, \alpha \in] 0,1[$. Let $\Omega$ be a bounded open connected subset of $\mathbb{R}^{n}$ of class $C^{k, \alpha}$. Let $\tilde{\phi} \in \mathcal{M}_{k, \alpha, j, m}$. Then, for all $u \in C_{0}^{k, \alpha}(\mathrm{cl} \Omega)$, the differential $\partial_{\phi_{\mid \phi=\phi}}\left(\Delta_{\phi} u\right)$ of the map of $C^{k, \alpha}\left(\mathrm{cl} \Omega, \mathbb{R}^{n}\right) \cap \mathcal{A}$ to $C^{k-2, \alpha}(\mathrm{cl} \Omega)$ that takes $\phi$ to $\Delta_{\phi} u$ at $\phi=\tilde{\phi}$ is delivered by the map

$$
\begin{aligned}
\psi \mapsto-2 \sum_{r, s=1}^{n}\left[\frac{\partial^{2}\left(u \circ \tilde{\phi}^{(-1)}\right)}{\partial y_{r} \partial y_{s}} \frac{\partial\left(\psi_{s} \circ \tilde{\phi}^{(-1)}\right)}{\partial y_{r}}\right] \circ \tilde{\phi}+ \\
-\sum_{s=1}^{n}\left[\frac{\partial\left(u \circ \tilde{\phi}^{(-1)}\right)}{\partial y_{s}} \Delta\left(\psi_{s} \circ \tilde{\phi}^{(-1)}\right)\right] \circ \tilde{\phi},
\end{aligned}
$$

for all $\psi \equiv\left(\psi_{1}, \ldots, \psi_{n}\right) \in C^{k, \alpha}\left(\operatorname{cl} \Omega, \mathbb{R}^{n}\right)$. Furthermore, if $u_{1}, u_{2}$ are two elements of $E_{\tilde{\phi}, j}$, then

$$
\int_{\Omega} \partial_{\phi \mid \phi=\tilde{\phi}}\left(\Delta_{\phi} u_{1}\right)(\psi) u_{2}|\operatorname{det} D \tilde{\phi}| d x=\int_{\partial \tilde{\phi}(\Omega)} \sum_{r=1}^{n}\left(\mu_{r} v_{r}\right) \frac{\partial v_{1}}{\partial v} \frac{\partial v_{2}}{\partial v} d \sigma,
$$

where $\mu \equiv\left(\mu_{1}, \ldots, \mu_{n}\right) \equiv \psi \circ \tilde{\phi}^{(-1)}, \quad v_{i} \equiv u_{i} \circ \tilde{\phi}^{(-1)}, \quad$ for $i=1,2$ and where $v \equiv\left(v_{1}, \ldots, v_{n}\right)$ denotes the unit exterior normal to $\partial \tilde{\phi}(\Omega)$.

Proof. Formula (3.43) can be derived by standard calculus in normed space. To prove formula (3.44), we note that by exploiting formula (3.43), by changing the variable in the integrals with the function $y=\phi(x)$, and by integrating by parts, we obtain

$$
\begin{aligned}
\int_{\Omega} \partial_{\phi \mid \phi=\tilde{\phi}}\left(\Delta_{\phi} u_{1}\right)(\psi) u_{2}|\operatorname{det} D \tilde{\phi}| d x=-\int_{\tilde{\phi}(\Omega)}\left(\Delta v_{1}\right) v_{2} \operatorname{div} \mu d y \\
\quad-\sum_{r=1}^{n} \int_{\tilde{\phi}(\Omega)} \frac{\partial v_{1}}{\partial y_{r}} \frac{\partial v_{2}}{\partial y_{r}} \operatorname{div} \mu d y+\sum_{r, s=1}^{n} \int_{\tilde{\phi}(\Omega)}\left(\frac{\partial \mu_{s}}{\partial y_{r}}+\frac{\partial \mu_{r}}{\partial y_{s}}\right) \frac{\partial v_{1}}{\partial y_{r}} \frac{\partial v_{2}}{\partial y_{s}} d y .
\end{aligned}
$$

Since $v_{1}$ and $v_{2}$ are eigenvectors of $-\Delta$ in $\tilde{\phi}(\Omega)$ relative to the eigenvalue $\lambda_{j}[\tilde{\phi}]$, the previous expression equals

$$
-\frac{1}{2} \int_{\tilde{\phi}(\Omega)}\left(\Delta\left(v_{1} v_{2}\right)\right) \operatorname{div} \mu d y+\sum_{r, s=1}^{n} \int_{\tilde{\phi}(\Omega)}\left(\frac{\partial \mu_{s}}{\partial y_{r}}+\frac{\partial \mu_{r}}{\partial y_{s}}\right) \frac{\partial v_{1}}{\partial y_{r}} \frac{\partial v_{2}}{\partial y_{s}} d y .
$$

Now, by exploiting the computations of Micheletti [18, pp. 161-163], one obtains that the expression in (3.46) equals the right-hand side of (3.44).

We are now ready to compute the derivative of $\lambda_{j}[\cdot]$ along $\gamma(\cdot)$ by using a standard argument. 
Theorem 3.47. Let $k \in \mathbb{N} \backslash\{0,1\}, j, m \in \mathbb{N} \backslash\{0\}, \alpha \in] 0,1[, \delta>0$. Let $\Omega$ be a bounded open connected subset of $\mathbb{R}^{n}$ of class $C^{k, \alpha}$. Let $\tilde{\phi}$ be an element of $\mathcal{M}_{k, \alpha, j, m}$. Let $\gamma \equiv\left(\gamma_{1}, \ldots, \gamma_{n}\right) \in C^{1}\left([-\delta, \delta], \mathcal{M}_{k, \alpha, j, m}\right), \quad \gamma(0)=\tilde{\phi} . \quad$ Let $u \in E_{\tilde{\phi}, j}$ be such that $<u, u>_{\tilde{\phi}}=1$. Then

$$
{\left.\frac{d}{d t}\right|_{t=0}} \lambda_{j}[\gamma(t)]=-\int_{\partial \tilde{\phi}(\Omega)} \sum_{r=1}^{n}\left(\gamma_{r}^{\prime}(0) \circ \tilde{\phi}^{(-1)}\right) v_{r}\left(\frac{\partial v}{\partial v}\right)^{2} d \sigma,
$$

where $v \equiv\left(v_{1}, \ldots, v_{n}\right)$ denotes the exterior unit normal to $\partial \tilde{\phi}(\Omega)$, and where $v \equiv u \circ \tilde{\phi}^{(-1)}$.

Proof. By possibly considering a restriction of $\gamma$, we can assume that $\gamma(t)$ belongs to the neighborhood $\mathcal{W}$ of $\tilde{\phi}$ of Theorem 3.34 , for all $t \in[-\delta, \delta]$. To shorten our notation, we set $\lambda(t) \equiv \lambda_{j}[\gamma(t)], p(t) \equiv \hat{p}_{\tilde{\phi}, j}[\gamma(t)]$, and we denote by a dot the differentiation with respect to $t$. Then we have $\Delta_{\gamma(t)} \circ p(t)+\lambda(t) p(t)=0$ in $\mathcal{L}\left(E_{\tilde{\phi}, j}, C^{k-2, \alpha}(\mathrm{cl} \Omega)\right)$, for all $t \in[-\delta, \delta]$. By differentiating with respect to $t$, by setting $t=0$, and by computing both sides of the resulting equation at $u \in E_{\tilde{\phi}, j}$, we obtain

$$
\Delta_{\tilde{\phi}}(\dot{p}(0)[u])+\lambda(0)(\dot{p}(0)[u])=-\left(\partial_{\phi \mid \phi=\tilde{\phi}}\left(\Delta_{\phi} u\right)[\dot{\gamma}(0)]\right)-\dot{\lambda}(0) u .
$$

Thus, by Theorem 3.11 and by formula (3.44), we deduce the validity of formula (3.48).

ACKNOWLEDGements. This paper represents an extension of a part of the work performed by P. D. Lamberti in his 'Laurea' Thesis under the guidance of M. Lanza de Cristoforis.

\section{REFERENCES}

1. M. S. Berger, Nonlinearity and functional analysis (Academic Press, 1977).

2. Sh. N. Chow and J. K. Hale, Methods of bifurcation theory (Springer-Verlag, 1982).

3. R. Courant and D. Hilbert, Methods of mathematical physics, Vol. I (Interscience, 1953).

4. S. J. Cox, The generalized gradient at a multiple eigenvalue, J. Functional Analysis 133 (1995), 30-40.

5. F. Desaint and J. P. Zolésio, Sensitivity analysis of all eigenvalues of a symmetrical elliptic operator, in Boundary control and variation, Lecture Notes in Pure and Applied Mathematics, 163 (1994), 141-160.

6. F. Desaint and J. P. Zolésio, Dérivées par rapport au domaine des valeurs propres du laplacien, C. R. Acad. Sci. Paris Sér. I Math. 321 (1995), 1337-1340.

7. E. W. Gekeler, On the perturbed eigenvalue problem, J. Math. Anal. Appl. 191 (1995), 540-546.

8. D. Gilbarg and N. S. Trudinger, Elliptic partial differential equations of second order (Springer-Verlag, 1983).

9. D. Henry, Perturbation of boundary value problems of partial differential equations, manuscript (Brasilia, 1994).

10. Q. Kong, H. Wu and A. Zettl, Dependence of eigenvalues on the problem, Math. Nachr. 188 (1997), 173-201.

11. P. D. Lamberti, Alcuni risultati relativi alla dipendenza degli autovalori del Laplaciano dal dominio, Tesi di Laurea (Univesità di Padova, 1998). 
12. M. Lanza de Cristoforis, Properties and pathologies of the composition and inversion operators in Schauder spaces, Rend. Acc. Naz. delle Sci. detta dei XL, XV (1991), 93-109.

13. M. Lanza de Cristoforis, Higher order differentiability properties of the composition and of the inversion operator, Indagationes Math. N. S. 5 (1994), 457-482.

14. M. Lanza de Cristoforis, A functional decomposition theorem for the conformal representation, J. Math. Soc. Japan 49 (1997), 759-780.

15. D. Lupo and A. M. Micheletti, On multiple eigenvalues of selfadjoint compact operators, J. Math. Anal. Appl., 172 (1993), 106-116.

16. D. Lupo and A. M. Micheletti, A remark on the structure of the set of perturbations which keep fixed the multiplicity of two eigenvalues, Revista de Matemáticas Aplicadas 16 (1995), 47-56.

17. D. Lupo and A. M. Micheletti, On the Persistence of the Multiplicity of Eigenvalues for Some Variational Elliptic Operator Depending on the Domain, J. Math. Anal. Appl. 193 (1995), 990-1002.

18. A. M. Micheletti, Perturbazione dello spettro dell'operatore di Laplace in relazione ad una variazione del campo, Annali della Scuola Normale di Pisa 26 (1972), 151-169.

19. A. M. Micheletti, Perturbazione dello spettro di un operatore ellittico di tipo variazionale in relazione ad una variazione del campo, Annali Mat. Pura Appl. 97 (1973), 267281.

20. J. Nečas, Les méthodes directes en théorie des équations elliptiques (Masson Paris, Academia, Prague, 1967).

21. G. Prodi, Dipendenza dal dominio degli autovalori dell'operatore di Laplace, Istituto Lombardo (Rend. Sc.) 128 (1994), 3-18.

22. G. Prodi and A. Ambrosetti, Analisi non lineare (Editrice tecnico scientifica, Pisa, 1973).

23. G. M. Troianiello, Elliptic differential equations and obstacle problems, The University Series in Mathematics (Plenum Press, 1987). 